\title{
PURE-COMPLETE SUBGROUPS OF DIRECT SUMS OF PRÜFER GROUPS
}

\author{
by PAUL HILL $\dagger$
}

(Received 9 March, 1970)

Suppose that $G$ is a $p$-primary abelian group. The subgroup $G[p]=\{x \in G: p x=0\}$ is called the socle of $G$ and any subgroup $S$ of $G[p]$ is called a subsocle of $G$. If each subsocle of $G$ supports a pure subgroup, then $G$ is said to be pure-complete [1]. It is well known that, if $G$ is a direct sum of cyclic groups, then $G$ is necessarily pure-complete. Further results about pure-complete groups are contained in [1] and [3].

By the Prüfer group (associated with the prime $p$ ), we mean the $p$-primary group generated by $a_{0}, a_{1}, \ldots, a_{n}, \ldots$ with defining relations: $p a_{0}=0$ and $p^{n} a_{n}=a_{0}$ for $n \geqq 1$. Suppose that $G$ is a subgroup of an arbitrary direct sum of Prüfer groups. Then $G$ is not too far removed from a direct sum of cyclic groups, for $G$ is an extension of an elementary group by a direct sum of cyclic groups. Thus it would be natural to ask the following question. Must $G$ be pure-complete (if it has no elements of infinite height)? The answer is in the negative; in fact, I proved in [2] that there exists a subgroup $G$ of a direct sum of Prüfer groups such that:

(1) $G$ has no elements of infinite height,

(2) $G$ is not a direct sum of cyclic groups,

(3) $G$ is not pure-complete.

At the time, little did I suspect that (2) implies (3) (whenever $G$ is a subgroup of a direct sum of Prüfer groups). The object of this paper is to prove that this is the case.

THEOREM. Suppose that $G$ is a subgroup of a direct sum of Prüfer groups. If $G$ is purecomplete, then $G$ is a direct sum of cyclic groups.

Proof. Let $G$ be a subgroup of a direct sum $K$ of Prüfer groups and suppose that $G$ is pure-complete. Letting $S=G \cap p^{\omega} K$, we have that $p S=0$ and that $G / S \cong\left\{G, p^{\omega} K\right\} / p^{\omega} K \subseteq$ $K / p^{\omega} K$ is a direct sum of cyclic groups. We know [4] that $G[p] / S \subseteq(G / S)[p]$ is a summable subsocle of $G / S$. Hence $G[p] / S=\sum_{n<\infty} T_{n}$, where the nonzero elements of $T_{n}$ have height exactly $n$ in $G / S$. We can write $T_{n}=\sum_{i \in Y_{(n)}}^{n}\left\{x_{i}+S\right\}$, and we can choose $x_{i}$ to have height $n$ in $G$ for each $i \in I(n)$. We now observe that

$$
G[p]=S+\sum_{n<\infty}\left(\sum_{i \in I(n)}\left\{x_{i}\right\}\right)
$$

is a natural decomposition of $G[p]$; see [4]. Since $G$ is pure-complete, there exists a pure subgroup $H$ of $G$ such that $H[p]=S$. And there is a pure subgroup $A$ of $G$ such that $A[p]=\sum\left\{x_{i}\right\}$ (whether $G$ is pure-complete or not). Since $(D)$ is a natural decomposition, $A+H$ is pure in $G$ and we have $G=A+H$. However, $G / S \cong A+p H$, which implies that $p H$, as well as $A$, is a direct sum of cyclic groups. It follows that $H$ is a direct sum of cyclic groups and so is $G$; see, for example, [6, p. 92].

† This work was supported by National Science Foundation Grant GP 12318. 
An immediate corollary to the theorem is the following.

COROLlaRY. If $G_{1}$ and $G_{2}$ are subgroups of a direct sum of Priffer groups, then $G=G_{1}+G_{2}$ is pure-complete if and only if $G_{1}$ and $G_{2}$ are pure-complete.

The corollary is false if the hypothesis that $G_{1}$ and $G_{2}$ are subgroups of a direct sum of Pruifer groups is deleted [1].

In conclusion, we remark that the above theorem points out the preponderance of groups without elements of infinite height having fairly simple structure that are not pure-complete. The reader may wish to compare this result with the first example [5] of a group without elements of infinite height and not pure-complete, as well as with the results of [3] on purecomplete groups.

\section{REFERENCES}

1. P. Hill, Pure subgroups having prescribed socles, Bull. Amer. Math. Soc. 71 (1965), 608-609.

2. P. Hill, Primary groups with uncountably many elements of infinite height, Arch. Math. 19 (1968), 279-283.

3. P. Hill and C. Megibben, On primary groups with countable basic subgroups, Trans. Amer. Math. Soc. 124 (1966), 49-59.

4. P. Hill and C. Megibben, On direct sums of countable groups and generalizations, Studies on abelian groups (Springer-Verlag, 1968).

5. C. Megibben, Note on a paper of Bernard Charles, Bull. Math. Soc. France 91 (1963), 453-454.

6. L. Fuchs, Infinite abelian groups, Vol. 1 (London, 1970).

Florida State University

Tallahassee, Florida 32306 